\title{
Polysèmes
}

Revue d'études intertextuelles et intermédiales

La défamiliarisation du quotidien ou l'amplification de l'ordinaire dans The Mezzanine (1986) de Nicholson Baker

\section{Claire Fabre}

\section{OpenEdition}

Journals

Édition électronique

URL : http://journals.openedition.org/polysemes/1766

DOI : 10.4000/polysemes.1766

ISSN : 2496-4212

Éditeur

SAIT

Édition imprimée

Date de publication : 2 janvier 2007

Pagination : 119-129

ISSN : 0999-4203

\section{Référence électronique}

Claire Fabre, «La défamiliarisation du quotidien ou l'amplification de l'ordinaire dans The Mezzanine (1986) de Nicholson Baker », Polysèmes [En ligne], 9 | 2007, mis en ligne le 01 janvier 2007, consulté le 19 avril 2019. URL : http://journals.openedition.org/polysemes/1766 ; DOI : 10.4000/polysemes. 1766

Ce document a été généré automatiquement le 19 avril 2019.

Polysèmes 


\title{
La défamiliarisation du quotidien ou l'amplification de l'ordinaire dans The Mezzanine (1986) de Nicholson Baker
}

\author{
Claire Fabre
}

1 L'attention à l'ordinaire n'est pas nouvelle dans les lettres américaines. Pour le philosophe Stanley Cavell, elle fut inaugurée par Emerson et Thoreau qui souhaitaient tout particulièrement prendre en compte (et célébrer) l'instant présent, par essence insaisissable. Cette volonté n'était nullement superflue dans leur projet littéraire mais bien l'un des devoirs essentiels de l'écrivain : « The everyday is what we cannot but aspire to, since it appears to us as lost on us. [...] There is nothing beyond the succession of each and everyday; and grasping a day, accepting the everyday, the ordinary, is not a given, but a task $»^{1}$. Nicholson Baker n'est pas éloigné de ces thèses lorsque, avec une désinvolture indéniable, il affirme : «I want the books to be about things that you don't notice when you're noticing them. You kind of notice them in passing, and never put a frame around them - and then somebody like me comes along and writes a book about them. And then that book itself becomes the frame $»^{2}$.

2 Ne rien perdre, tout embrasser dans et par l'écriture : telle parait être aussi l'entreprise du narrateur de The Mezzanine (1986) ${ }^{3}$ qui, en se remémorant sa course sur l'escalator ce jour-là (un jour situé dans un passé relativement proche, mais indéterminé : « on sunny days like this one ", 3) relate ses pensées et ses observations dans leurs moindres détails et rend ainsi visibles les choses les plus petites que caresse son regard. Rien ne peut se soustraire à cette mise en mots, cette emprise des mots sur le monde tant son projet semble aspirer à l'exhaustivité. Comme l'a montré Véronique Béghain ${ }^{4}$, Baker s'inscrit dans la tradition des écrivains américains qui se proposent de renouveler le regard porté sur le quotidien en le défamiliarisant. 
On peut alors se demander comment s'opère cette défamiliarisation du quotidien, en se concentrant surtout sur les objets que le roman met en circulation. The Mezzanine est en effet peuplé d'objets : ceux qui entourent directement le narrateur (l'escalator, ses lacets de chaussures, les toilettes de son entreprise) mais aussi tous ceux qui sont convoqués au cours des descriptions par associations d'idées, analogie, métaphore, comparaison... Il en résulte un monde foisonnant d'objets hétéroclites, de toutes les formes et tailles, dont la description est au cœur du roman. Il va sans dire qu'un tel projet inverse la traditionnelle suprématie du narratif sur le descriptif, sans toutefois éliminer entièrement le premier. Philippe Hamon a bien montré que le fait était caractéristique de la littérature contemporaine, à tel point que chez certains écrivains, le narratif semblait réduit à une fonction de prétexte ou de faire-valoir du descriptif 5 .

Cependant, dans The Mezzanine, les modalités d'apparition du descriptif sont étroitement liées à celles du narratif si bien qu'il s'avère difficile, voire impossible de les distinguer nettement. La dimension heuristique de la quête du narrateur se fait jour dès que l'on observe cet enchevêtrement: la "dissection $»^{6}$ obsessionnelle à laquelle il soumet le quotidien est assortie de considérations qui dépassent largement les contours de l'objet et qui lui permettent de fonder une multitude de micro-théories personnelles dans lesquelles se combinent naïveté, loufoquerie et tendresse. Il peut alors articuler son histoire personnelle à des développements historiques plus larges. Aussi le descriptif permet-il des changements d'échelle, notion centrale pour décrire les stratégies de défamiliarisation qui sont au cœur de l'œuvre. Tous les niveaux du texte sont affectés par ces variations d'échelle - l'espace, le temps, les perceptions, les analogies entre objets qui ont généralement comme dénominateur commun le processus d'amplification.

Le ton qui domine le descriptif dans le récit n'est pas étranger aux effets comiques, voire burlesques du roman : s'il n'est nullement austère ou scientifique, il peut parfois parodier le didactisme de la description sérieuse. Au-delà de sa fonction heuristique pour le narrateur, le descriptif est bien chez Baker le lieu privilégié d'une expression lyrique.

\section{Narratif vs descriptif ?}

6 Résumer l'intrigue centrale (si l'on peut encore parler d'intrigue) de The Mezzanine ne devrait pas prendre plus de quelques secondes : un employé remonte à son bureau, situé sur la mezzanine de l'immeuble, après sa pause déjeuner, en tenant à la main le petit sac en papier du magasin CVS contenant des lacets de chaussure neufs destinés à remplacer les précédents qui ont cédé le matin même. Mais à l'intérieur de ce cadre diégétique étroit, rigoureusement respecté jusqu'à la fin du roman, se glissent de nombreuses réflexions et observations sous forme de digressions de taille très variable et qui sont incluses, soit dans ce que l'on a coutume d'appeler le "texte principal», soit dans les notes de bas de page. Si l'on est en devoir de prendre des précautions concernant la terminologie typographique, c'est que l'une des caractéristiques de The Mezzanine consiste à inverser les proportions entre texte et notes, comme on le voit au milieu du roman où il ne subsiste plus que quatre lignes de texte en haut de page, le reste étant occupé par la note, et ce sur pas moins de quatre pages ${ }^{7}$. Pour l'heure, relevons surtout que ce dispositif contribue à subvertir non seulement la hiérarchie entre le descriptif et le narratif, mais également à brouiller les frontières entre les deux. 
7 Dans The Mezzanine, la digression descriptive ne peut en aucun cas être appelée statique à cause du rôle d'embrayeur narratif qu'elle endosse souvent. C'est ainsi que tout au long du récit, et le plus souvent dans les notes de bas de page, sont distillés incidemment, et à dose homéopathique, des éléments de l'histoire personnelle du narrateur, comme ses rapports avec sa compagne, L., ou ses souvenirs d'enfance avec ses parents.

Par exemple, au détour d'une réflexion sur les factures de cartes de crédit, il évoque un dîner avec L., sa compagne, qui n'apparaît que sur ce mode minimaliste et sporadique dans quelques notes. De même, son développement sur l'évolution des poignées de porte dans les habitations américaines lui donne l'occasion de décrire l'admiration qu'il porte à son père (à cause des cravates que ce dernier avait coutume de suspendre aux poignées de portes), et de donner, en réduction, le récit des étapes de son accession à la maturité. L'admiration qu'il portait, enfant, à sa mère, s'exprime à travers la remémoration d'un geste quotidien précis : la manière dont elle était capable de retourner un pull («inside out ») en un tournemain. En revanche, plus loin dans le récit, il manifeste son agacement à l'égard de sa mère devant son incapacité à s'adapter aux nouveaux systèmes d'ouverture des cartons de lait, détail qui est inséré dans une longue note historique concernant l'évolution de ces systèmes d'ouverture. Des bribes de l'histoire intime du narrateur se glissent ainsi dans le récit, pour livrer quelques vagues contours de son existence.

Le texte offre ainsi une dynamique complexe: le narratif, malgré les tentatives du narrateur pour en réduire la part, ressurgit à la faveur des descriptions comme s'il résistait à toute marginalisation définitive. C'est d'ailleurs la même contradiction qui semble animer le narrateur qui affirme à plusieurs reprises - ce qui laisse naturellement soupçonner une part de dénégation - ne pas vouloir succomber à la nostalgie lorsqu'il se laisse emporter par le flux de ses souvenirs. Par exemple, après la longue évocation des différentes formes de conditionnement du lait - métonymie implicite de l'enfance - il affirme vouloir se prémunir contre les dangers d'un tel regard rétrospectif :

All of these nostalgia-driven memories pour out of that Sealtest carton, pulling me off course, distorting what I want to be a simple statement of gratitude for a great packaging design that happened to come into widespread use when I was little. I look forward to the time when I will have thought about milk and cheese products enough as an adult that the unpasteurized taint of sentimentality will lift from the subject. (46)

10 Ainsi se dessine le rêve - utopique - d'un descriptif entièrement débarrassé de narratif, que le texte réfute dans son ensemble. L'exemple des cartons de lait est paradigmatique de tous ces autres descriptions-inventaires historiques. N'échappent pas à sa plume: l'historique des pailles, des machines à café, des distributeurs de serviettes en papier dans les toilettes publiques... Si ces objets de l'environnement du narrateur commencent tous par être décrits, ils cèdent bien vite la place à ceux qui les précèdent dans son souvenir. En d'autres termes, l'objet que l'on s'attendrait à voir décrit en détail n'est que le support de tous les autres (à la fois semblables et différents) qu'il convoque, et l'énumération tient lieu de description. Ainsi la digression descriptive est-elle l'instrument d'une expansion spatiale et temporelle du récit car elle permet d'évoquer des pans entiers de l'histoire des États-Unis (qui se résume à celle de ses artefacts), ou de l'histoire personnelle du narrateur.

11 Au centre exact du roman se trouve la plus longue note de bas de page où l'on trouve une de ces listes. La note est appelée par le souvenir d'un jour où le narrateur avait 
délibérément emprunté des escalators avec ses lacets défaits, pour prouver et se prouver que ceux-ci ne présentaient aucun danger :

And escalators are safe: their safety the result (I now believe) of a brilliant decision to groove the surfaces of the stairway so that they mesh perfectly with the teeth of the metal comblike plates at the top and bottom, making it impossible for stray objects, such as coins or shoe-lace-ends, to get caught in the gap between the moving steps and the fixed floor. I gave no direct thought to the escalator's grooves that afternoon, and indeed at that time I had distinct notions as to their purpose: I thought they were for traction, or possibly were purely decorative; grooved to remind us of how beautiful grooved surfaces are as a class: the grooves on the underside of the blue whale that must render some hydrodynamic or thermal advantage; the grooves left by a rake in loose soil or by a harrow in a field; the single groove that a skater's blade makes in the ice; the grooves in socks that allow them to stretch, and in corduroy down which you can run your ballpoint pen; the grooves of record.

During the period that I rode the escalators with untied shoelaces, I spent the winters speed-skating (an escalator step, incidentally, looks like a row of upturned skate blades) around and around an outdoor pond behind old Italian skaters with raisin faces and hooded sweatshirts who held their skate guards behind their backs and moved with long, slow unvarying strokes; and the summers I spent listening to records. (65)

Dans ce passage, l'émerveillement devant l'objet quotidien dont parle Tony Tanner ("wonder ») est thématisé par le narrateur lui-même, et son discours est émaillé d'expressions qui expriment son admiration devant un détail : la perfection formelle des rainures de l'escalator : "brilliant », « they mesh perfectly ", «beautiful ». Mais bien vite cette description cède le pas à une énumération enthousiaste grâce à une notation métadescriptive : « to remind us of how beautiful grooved surfaces are 'as a class' ». S'ensuit un catalogue lyrique à la Whitman, musicalement rythmé par l'anaphore doublée d'un jeu de mots («the grooves ») qui juxtapose des objets (ou des animaux) aux tailles très diverses - de la baleine au patin à glace. L'impertinence parodique pointe naturellement dans cette mention de la baleine qui n'est plus le support d'infinies extrapolations métaphoriques comme chez Melville mais qui est prise elle-même, à titre d'objet, dans un réseau plus vaste d'analogies dans laquelle la baleine joue ici un rôle de signe métadescriptif. Les rainures, qui n'étaient au départ qu'un détail, sont devenues en quelque sorte la raison - au sens mathématique du terme - de cette suite apparemment hétéroclite. Dans un deuxième temps, le texte se chargera de réutiliser certains éléments de la liste (le patin à glace, les disques) pour les inscrire dans une deuxième chaîne signifiante, narrative cette fois.

13 Si l'on perçoit une volonté d'exhaustivité dans The Mezzanine, ce n'est pas tant pour épuiser le même objet, que pour établir le plus grand nombre de relations possibles entre les objets. Cette ambition est d'ailleurs corroborée par une microthéorie que le narrateur a reçue en héritage de sa mère sur l'importance relative du nombre de synapses par rapport au nombre de neurones dans le cerveau :

It's true, she said, "that your individual brain cells are dying, but the ones that stay grow more and more connections, and those connections keep branching out over the years, and that's the progress you have to keep in mind. It's the number of links that are important, not the raw number of cells."

14 Et le narrateur de mettre aussitôt la théorie en application en formant à son tour plusieurs théories en relation avec celle-ci. Cette découverte donnée dans la liste de ses avancées vers la maturité par le narrateur est essentielle pour comprendre l'un des 
modes fondamentaux de production du texte, et plus encore l'essence de la défamiliarisation du quotidien chez Baker : ce n'est pas que l'objet ou le geste familier se mette tout à coup à présenter une facette étrange mais c'est bien sa mise en relation avec d'autres, de taille et de nature très différentes, qui crée un effet d'incongruité.

\section{Changements d'échelle : l'amplification}

Parce qu'il porte son regard sur des objets infimes, on dira volontiers que Nicholson Baker est un miniaturiste, mais comme le montre l'exemple des rainures, le texte effectue également un mouvement du plus petit vers le plus grand. Bachelard a montré que la miniature était paradoxale parce qu'elle permettait l'inclusion du plus grand dans le plus petit ${ }^{9}$. En effet, selon Bachelard, la miniature recrée non seulement une portion du monde à petite échelle, mais par métonymie, le monde dans son ensemble, propriété qu'il nomme la «cosmicité de l'image ». Lorsqu'il est pris dans la logique de l'énumération, l'objet de Baker se différencie toutefois de cette définition de la miniature en ce qu'il n'est nullement enfermant, mais semble au contraire ouvrir des voies de frayage vers d'autres objets. L'idée s'appliquerait davantage au texte lui-même, qui crée un cadre narratif minimal à l'intérieur duquel le descriptif permet une expansion virtuellement infinie.

Dans l'exemple précédent, l'énumération hétéroclite procède d'une variation de l'échelle des objets évoqués : l'escalator, la baleine, les sillons dans la terre, la trace du patin sur la glace et enfin, les micro-sillons du disque. L'escalator, dont Antoine Caze a très bien montré la valeur metatextuelle dans ce récit ${ }^{10}$, ne pourrait-il s'entendre comme une autre métaphore des processus à l'œuvre dans le texte, une sorte de version technologique de l'échelle, que l'on aurait affectée seulement d'un mouvement perpétuel?

17 La question de la taille relative des choses est un motif constant de l'œuvre de Baker, et elle est source de comique, comme dans son essai « The Size of Thoughts » qui se propose d'établir une échelle permettant de mesurer concrètement la taille des pensées : "Each thought has a size, and most are about three feet tall, with the level complexity of a lawnmower engine, or a cigarette lighter, or those tubes of toothpaste that, by mingling several hidden pastes and gels, create a pleasantly striped product ${ }^{11}$.

\section{Le descriptif comme lieu du figural}

On l'a vu, les descriptions permettent au narrateur d'établir des listes et des théories qui révèlent sa soif de savoir et son désir de le partager. En d'autres termes, elles ont une fonction heuristique liée au trajet initiatique du narrateur et participent d'un projet total, d'une utopie du «tout mettre en mots ». Mais le paradoxe de l'écriture de Nicholson Baker tient à une économie et une sobriété de la langue qui ne sont pas incompatibles avec une jouissance à pouvoir dire le monde, le décrire, le passer en revue.

La digression descriptive chez Baker se donne en effet comme l'espace d'une tension entre perception subjective et volonté de maîtrise sur le monde. En cela, elle exploite la différence que Philippe Hamon estime fondatrice du genre, entre description et définition. Certes, tant dans son lexique technique que dans sa pose assertive, la description chez Baker tend vers une certaine technicité caractéristique de la définition, mais ceci est contrebalancé par toute une série d'expressions qui modalisent le discours (comme les adverbes "probably», ou "possibly») et exhibent la part purement 
subjective des analogies. En fait, le descriptif dans The Mezzanine affiche souvent ses failles, son incomplétude, ses manques. C'est dans ce flottement que l'on perçoit la singularité de la voix : lieu d'une expression poétique, d'un regard singulier.

Comme l'a montré Ricœur, la métaphore est par excellence le lieu où se manifeste le regard subjectif: "Le 'voir-comme' offre le chaînon manquant dans la chaîne de l'explication ; le 'voir-comme' est la face sensible du langage poétique ; mi-pensée, miexpérience, le 'voir-comme' est la relation intuitive qui fait tenir ensemble le sens et l'image $»^{12}$. Si le narrateur de The Mezzanine utilise assez peu de métaphores construites et explicites, en revanche le métaphorique s'insère insidieusement dans l'ensemble du texte, sous couvert de précision descriptive, comme par exemple dans la description du simple geste qui consiste à ouvrir un sachet de pansement : "pulling on the red thread that is supposed to butterfly a Band-Aid and having it wrest free from the wrapper without tearing it ». L'image est ainsi camouflée par le déplacement syntaxique qui assure la transformation d'un nom en verbe, et l'émerveillement du narrateur demeure implicite, comme s'il était généreusement donné à déchiffrer, et donc à redécouvrir, au lecteur.

Par ailleurs, les comparaisons et métaphores participent aux stratégies d'expansion du texte car elles sont bien souvent prises dans des structures emboîtées grâce, notamment, au système complexe des notes de bas de pages.

Un dernier exemple me permettra d'illustrer la façon dont le métaphorique se glisse dans le descriptif et provoque une amplification du texte. Le passage est une note de bas de page, située à un moment du texte où le narrateur se remémore la première fois qu'il s'est laissé porter par un escalator (c'est-à-dire sans ajouter son propre mouvement à celui de l'engin). Il était, ce jour-là, chargé de paquets, et notamment d'une casserole de la marque Revere, ce qui lui inspire la note suivante :

In those first months of cooking for myself, after years of eating the food that Seiler's and ARA had cooked for me, I studied with fresh interest the origination of the boiling bubbles in the Revere pan as I waited to pour in the Ronzoni shells: at the very beginning of boiling, grains of mercury broke free and rose upward only from special points on the floor of the pan, requiring a little scratch or irregularity in the metal to harbor their change of phase; later, several beaded curtains of midsized spheres streamed where the parallel curves of the electric coil were most completely in contact with the pan's underside; later still, as glutinous, toad-like globes of hard boiling took over, my glasses misted - and I was reminded of being awakened by my parents earlier from dreams in which I had been trying to drink very thick shakes through impossibly slender straws. My father carried me to the bright kitchen saying cheerfully "Croup again, croup again", his hair sticking in unusual directions, and he held me near the plume of steam coming from the small kettle that my mother had put on. I inhaled, the desire to croak melted in the branches behind my sternum, and as I breathed, I thought happily about the blue gas flame pouring upward and flattening itself against the bottom of the kettle the same flame which a few years later I was allowed to cook hot dogs over, skewering them on a dinner fork: grease from the hot dogs was released in shortlived fiery sparks, best seen if you turned off the light, though notable too for their paler yellow effects in daylight, and the heat charred to prominence the spoked pattern of the two ends of the hot dog. Anyway, once I let my glasses clear, I poured the Ronzoni shells into the tumultuous water: there was a hiss and a moment of complete, white-watered calm.

Unless you stirred at that point, I found, your yield of shells would diminish, because some would stick to the bottom of the pan. (102) 
Ici, on peut remarquer avant toute chose la fusion entre descriptif et narratif qui repose notamment sur l'alternance entre les modes singulatif et itératif: le prétérit d'habitude (itératif) ("in those first months I studied») est à contraster avec le prétérit «I was reminded of being awakened» qui annonce le récit d'un événement particulier de l'enfance du narrateur. Le texte s'enchaîne selon le principe de l'emboîtement des souvenirs (une image en appelant une autre) dont les liens sont analogiques ou de contiguïté, sauf dans le cas de la flamme à la fin, qui est la même que celle sur laquelle il se faisait griller des hot dogs. Dans la texture même de cet extrait, des motifs insistants font osciller le texte entre l'observation scientifique et le paysage poétique. La rhétorique générale du passage ainsi que certains termes comme "grains of mercury" qui apparentent la cuisson des pâtes avec une expérience de chimie sont doublés d'une isotopie paysagère: on remarque la récurrence du crapaud ("toad-like" qui appelle ensuite « croak »); l'image des branches d'un arbre sert à décrire les poumons ; la surface de l'eau devient une mer agitée, etc. Bref, tout un paysage naturel, bien qu'évoqué par petites touches discrètes, est contenu dans cet épisode de la vie quotidienne.

Si l'on en croit William Gass, il n'y a pas de descriptions en fiction, seulement des "constructions $»^{13}$. Ainsi décrire l'objet revient-il à le désancrer du réel pour le réinscrire dans un nouveau système de signes qui possède sa propre logique, sa propre économie rhétorique.

Pour Baker, l'objet quotidien suscite descriptions et commentaires d'une longueur inversement proportionnelle à sa taille initiale. Pourtant, l'objet singulier semble échapper au regard toujours porté ailleurs, comme s'il demeurait fondamentalement indescriptible.

En définitive, chez Baker, rien n'est vraiment « donné à voir », si ce n'est une multitude de rapports entre les choses. Etablir des points de connexion, tracer des ensembles et des catalogues : telle est sa manière unique et singulière de se mouvoir dans le monde et les mots, et de "déréaliser » l'objet, malgré l'apparente saturation du référentiel dans le texte. L'amplification rejoint ici naturellement son sens rhétorique ( amplificatio $»^{14}$ ), c'est-à-dire l'art d'agrémenter le discours. Bien que les traités de rhétorique nous mettent en garde contre un usage abusif de ces éléments, de crainte de tomber dans une verbosité inélégante, Nicholson Baker, avec ses audaces et ses excès comiques joue sans cesse à effleurer cette limite.

Enfin, l'étude du descriptif dans The Mezzanine nous aura permis de mettre en valeur une tension essentielle du texte entre un discours de maîtrise sur le monde et l'aveu de lacunes, de failles qui forcent le narrateur à recourir à des images métaphoriques. Le descriptif comme discours de savoir et de maîtrise est à la fois convoqué et parodié, travaillé à la fois par le lyrisme et par la dérision.

\section{NOTES}

1. Stanley Cavell, In Quest of the Ordinary, Chicago: U of Chicago P, 1988, 171. 
2. Interview de 1994 accordée à Alexander Lawrence et David Strauss : « A Life in Detail».

3. Nicholson Baker, The Mezzanine, New York: Weidenfeld and Nicolson, 1986. Toutes les références entre parenthèses dans le cours de l'article renvoient à cette édition.

4. Véronique Béghain, "Accords et discords: l'œuvre comique de Nicholson Baker», dans Humoresques, $n^{\circ} 15$, «L'Humour américain », dir. Martine Chard-Hutchinson, Paris : Éditions de la Maison des sciences de l'homme, 2003.

5. Philippe Hamon donne l'exemple de Claude Simon, pour qui « [l]a description n'est donc plus un simple moment décoratif dans l'œuvre, mais le véritable 'moteur' de l'œuvre tout entière ", in La Description littéraire, Paris : Macula, 1991, 193.

6. Véronique Béghain, « Accords et discords : l'œuvre comique de Nicholson Baker », op cit., 7.

7. Les effets de cette inversion ont été très bien étudiés par A. Cazé et B. Félix (communications données au groupe de recherche LOLITA - Laboratoire Orléans-Tours de Littérature Américaine).

8. Véronique Béghain, «Accords et discords : l'œuvre comique de Nicholson Baker », op. cit., 7.

9. Gaston Bachelard, Poétique de l'espace, Paris : PUF, 1967.

10. Notamment parce qu'il condense les mouvements cycliques repérables à tous les niveaux du texte.

11. Nicholson Baker, The Size of Thoughts, New York: Vintage Books, 1997, 10.

12. Paul Ricœur, La Métaphore vive, Paris : Éditions du Seuil, 1975, 270.

13. William Gass, Finding a Form, New York: Cornell UP, 1996, 40.

14. "Les Anciens appelaient amplification le traitement du discours dans son ensemble [...] Il y fallait des descriptions, des comparaisons, des exemples, une discussion des raisons, du pathétique, des souvenirs, des citations de citoyens illustres ou des poètes, des explications, des justifications. Pour finir, une accumulation d'arguments, de faits, ou seulement de phrases, voire de mots synonymes. Telle est l'amplification oratoire, appelée encore, quand elle va trop loin, développement outré, pathétique, superflu, verbeux, diffus ", B. Dupriez, Gradus. Les Procédés littéraires, Paris : Union Générale d'Éditions, 1984.

\section{INDEX}

oeuvrecitee Mezzanine (The) 